\title{
La Myobank-AFM : une ressource précieuse pour la recherche
}

\author{
Stéphane Vasseur, Maud Chapart-Leclert
}

La Myobank-AFM de l'Institut de Myologie est un centre de ressources biologiques à but non lucratif travaillant dans le respect des droits fondamentaux de la personne et à l'usage exclusif de la recherche scientifique. Créée en 1996 par l'AFM-Téléthon pour permettre aux équipes de chercheurs de travailler directement sur des ressources biologiques humaines, cette banque est maintenant un service dépendant de l'Association Institut de Myologie (AIM). La Myobank-AFM a pour vocation de favoriser le développement de la recherche au bénéfice des personnes atteintes de maladies neuromusculaires par le biais d'activités de collecte de tissus musculaires et d'autres ressources biologiques d'origine humaine.

Sont collectés principalement :

- les résidus opératoires recueillis au cours d'une intervention chirurgicale; provenant soit de personnes atteintes de maladies neuromusculaires, soit de volontaires sains fournissant ainsi du matériel témoin ;

- les surplus de biopsies musculaires, si par exemple, l'intégralité du fragment n'a pas été utilisé pour l'établissement d'un diagnostic ;

- des fluides biologiques (sérum, plasma, autres) recueillis à l'occasion de prélèvements déjà programmés dans le cadre de la prise en charge médicale courante.

Dans tous les cas, la conservation de tels échantillons fait l'objet d'une demande de consentement de la personne concernée.

Les pathologies principalement collectées à la Myobank-AFM sont la dystrophie musculaire de Duchenne, l'amyotrophie spinale infantile, la dystrophie musculaire facioscapulohumérale mais aussi des maladies neuromusculaires plus rares intéressant tout autant les chercheurs. Les échantillons correspondant à des individus témoins sont également numériquement et stratégiquement importants.

Stéphane Vasseur Maud Chapart-Leclert Myobank-AFM, Institut de Myologie, Paris, France

Contact

s.vasseur@institut-

myologie.org
Pour ce qui concerne la conservation de cultures immortalisées de fibroblastes ou de myoblastes, la Myobank-AFM collabore étroitement avec le plateau technique Myoline du Centre de Recherche en
Myologie (Sorbonne Université-Inserm UMRS974) et avec la Banque du Généthon.

\section{Les préparations}

La préparation et la conservation des échantillons renvoient à des savoir-faire techniques et à des bonnes pratiques professionnelles mises en œuvre pendant près de trente ans. La préparation des échantillons (transformation) se fait selon la demande des chercheurs, en fonction de l'utilisation qui en est prévue, de façon à maintenir les qualités intrinsèques du tissu. Les différentes préparations réalisées par la Myobank-AFM permettent la culture cellulaire (acheminement à $4^{\circ} \mathrm{C}$ en milieu spécifique), la mise en culture à partir de tissus congelés (cryoconservation en milieu spécifique), les études biochimiques (congélation directe à l'azote), les études nécessitant le maintien de la morphologie (congélation dans l'isopentane), les études d'ARN (préparation en milieu spécifique RNA-Later ${ }^{\circledR}$ ). Concernant les fluides, une préparation est réalisée pour recueillir du plasma/sérum principalement.

\section{Le stockage et la mise à disposition}

Les ressources biologiques sont conservées dans des enceintes de froid à $-80^{\circ} \mathrm{C}$ et des cuves d'azote à - $196^{\circ} \mathrm{C}$. Les enceintes sont surveillées par un système d'alarme et de relevés de température H24. Une astreinte technique est assurée par l'équipe.

Pour bénéficier des services de la Myobank-AFM, les équipes de recherche font une demande écrite explicitant leur besoin en rapport à leur thématique de recherche. En même temps, par une convention signée et/ou un contrat, le chercheur s'engage à : respecter la législation en vigueur pour l'utilisation des échantillons biologiques humains dans le cadre de recherches scientifiques, utiliser uniquement ces échantillons pour les recherches scientifiques décrites dans sa demande, ne pas prêter ni céder au profit d'un tiers les échantillons obtenus, ne pas utiliser les échantillons dans un but lucratif, signaler l'arrêt du projet de recherche, citer Myobank-AFM de l'Institut de Myologie dans les remerciements des publications qui présentent des résultats obtenus grâce à l'utilisation des ressources biologiques mises à dispositions par Myobank (cf. charte et demande de ressources biologiques"). 
Le service est ouvert à toute demande émanant d'équipes de recherche, qu'elles soient en France ou à l'étranger. Chaque demande est examinée par l'équipe et transmise pour avis au conseil scientifique de Myobank composé d'un clinicien de l'Institut de Myologie (Anthony Béhin), du directeur du Centre de Recherche en Myologie de l'Institut de Myologie (Bertrand Fontaine) et du directeur scientifique de l'AFM-Téléthon (Serge Braun). Une fois obtenu le feu vert du conseil scientifique, la Myobank-AFM fournit dans les meilleurs délais les échantillons demandés par les chercheurs. Si elle n'en dispose pas en stock, elle peut s'appuyer sur son réseau de professionnels de santé pour les collecter de manière prospective (Figure 1).
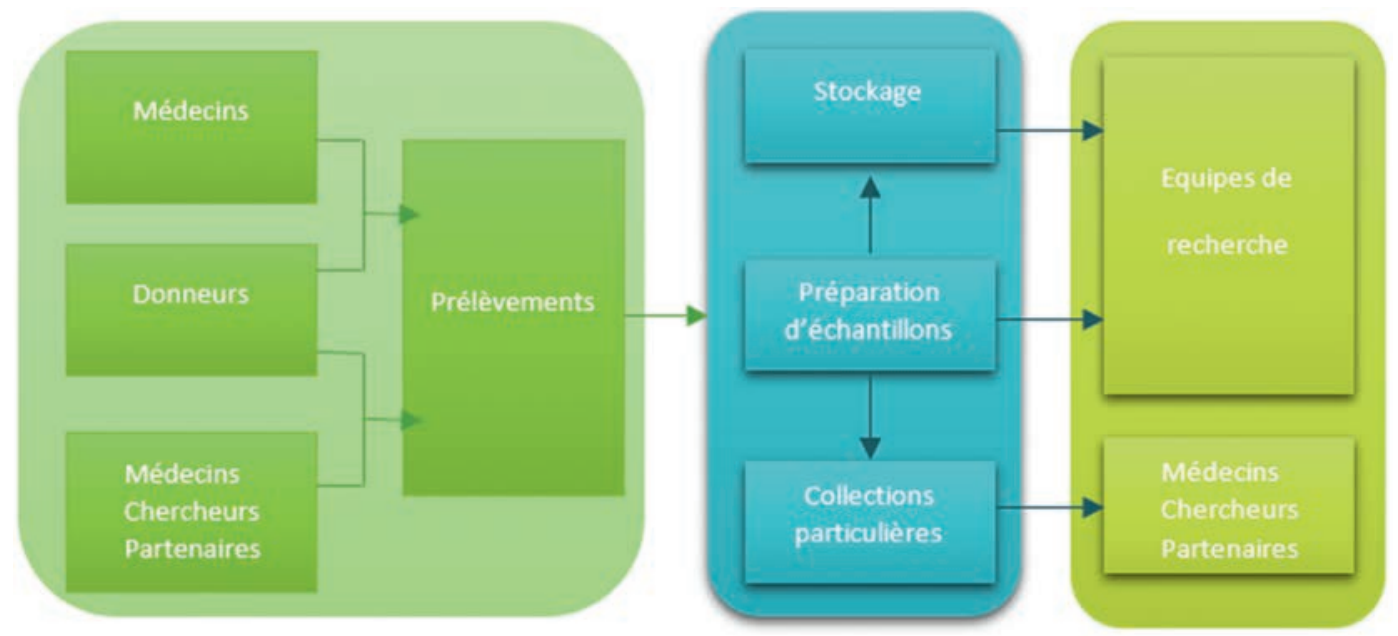

Figure 1

Schéma d'organisation de la Myobank-AFM.

La mission de la Myobank-AFM (https://www.institut-myologie.org/recherche/myobank/) est de recueillir, préparer, stocker et distribuer des ressources biologiques humaines, à l'usage exclusif de la recherche scientifique. Elle assure : le respect des règles de bonne pratique de laboratoire, le respect de protocoles validés et d'une méthodologie de qualité, le respect des contrôles de sécurité (température, incendie, malveillance, sauvegarde des données), la traçabilité des échantillons par la base de données $\mathrm{GBC}^{\circ}$ (Gestion Banque et Collection), développée conçue pour assurer une gestion rigoureuse des fichiers informatisés pour ses activités.

Ces dernières années, Myobank a également apporté sa contribution au bon déroulement d'essais

de thérapie génétique dans la dystrophie musculaire de Duchenne (essais Essence, Wave et GNT016 micro-dystrophine), dans la myopathie centronucléaire (étude DYN101) et dans la myopathie myotubulaire liée à l'X (essai Aspiro).

La Myobank-AFM conserve actuellement près de 90000 échantillons et reçoit une trentaine de demandes de chercheurs par an.

À ce titre, et depuis sa création, elle a été remerciée dans plus de 190 articles de différents journaux scientifiques.

Myobank-AFM: a valuable resource for research

\section{LIENS D'INTÉRÊT}

Les auteurs déclarent n'avoir aucun lien d'intérêt concernant les données publiées dans cet article. 